\title{
Wireless Robotic ARM
}

\section{AbhishekVerma(B.Tech, CS Engg.), Ashish Sharma(B.Tech, EL Engg.)}

\begin{abstract}
Robots are now becoming a part of our daily life and performing everyday tasks much more efficiently and smartly. Robotics as a branch of engineering deals with design, construction, operation, and application of robots. Their mobility is as efficient as the control signal. Humans can perform highly precise tasks without much effort. This project aims at giving the same mobility to robotic arm, so that it can perform tasks precisely in an environment not accessible or suitable for humans, remotely using very simple potentiometers as sensors and Atmel ATmega32 microcontroller.
\end{abstract}

Keywords: Potentiometer, Atmel, ATmega32, AVR, Haptic Hand, Wireless Hand

\section{Introduction}

A robot is usually an electro-mechanical machine that is guided by means of computer and electronic programming. Robots can be autonomous, semi-autonomous or remotely controlled. Robots can be used for wide variety of tasks ranging from house hold tasks, industrial applications to exploration of outer space.

Now days, advanced robots consist of hardware along with software. Software provide the necessary signal of intelligence to guide the mechanical parts to perform task. Humans have the extraordinary capability of haptic - sense of touch. Our system exploit this feature to provide precise control signals to mechanical robotic arm wirelessly, giving ability to perform even very delicate tasks without much effort.

\section{Design of the Robotic ARM}

The wireless robotic arm mainly consists of Atmel ATmega32 microcontroller and potentiometers as controlling device. The actuators are 9v DC motor. The whole system works on principle of interfacing the potentiometer with microcontroller processing the signal from the potentiometer and giving output to motors so as to move the mechanical arm. The motors are interfaced with the help of wireless circuit (RF). It consist of a wireless RF transmitter and RF receiver.

Potentiometers are fixed to a wearable remote control. Potentiometer moves when remote moves. The potentiometer converts the mechanical motion into electrical motion. Hence, on the motion of the remote the potentiometers produce the electrical pulses, which are then route to microcontroller. The pulse are analogue signal which need to be converted to digital signal so that it can work according to programming. For this the onboard ADC (Analogue to Digital Converter) of Atmega32 microcontroller is used.

The whole project mainly consist of following parts:

1. Robotic ARM

2. Microcontroller

3. Remote control

Let's look at each part in detail.

\section{Robotic ARM}

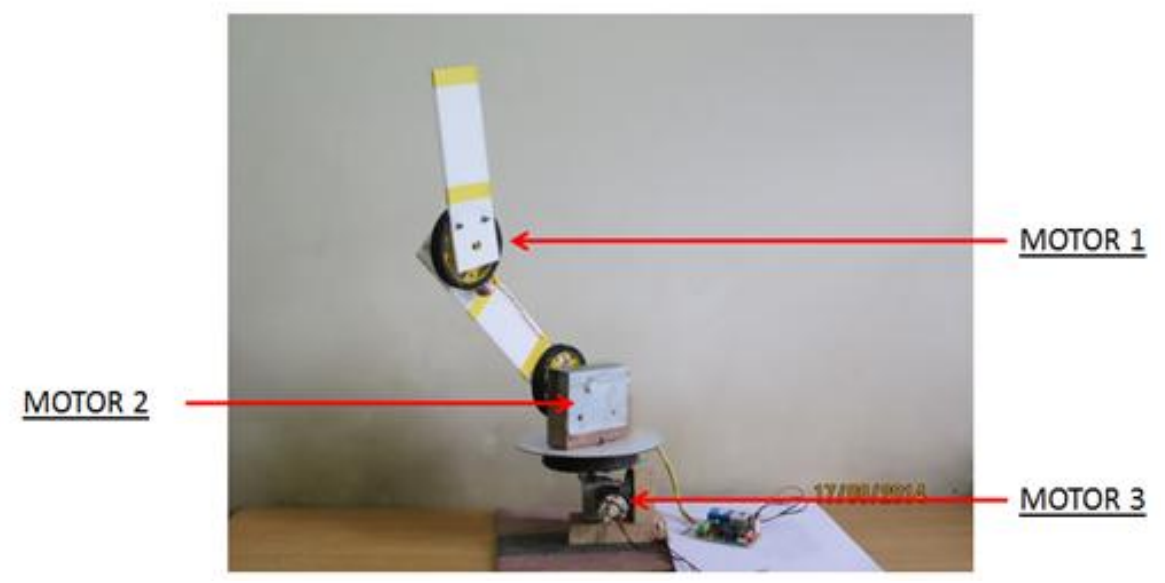


The ARM consist of 3 motors.

MOTOR 1 for wrist movement.

MOTOR 2 for elbow movement.

MOTOR 3 for rotational motion (Controlled manually).

Motors used here in this project are 9V DC Geared motors. Motors are connected to RF receiver with a motor driving IC- L293D. Motor driving IC is needed because the digital circuit works on 5V logic voltage supply and to drive motors we need $9 \mathrm{v}$ and high current power source. Thus to combine control signal and voltage supply we need a switching circuit, and this is achieved with help of motor driving IC.

\section{Microcontroller - Atmel ATmega32}

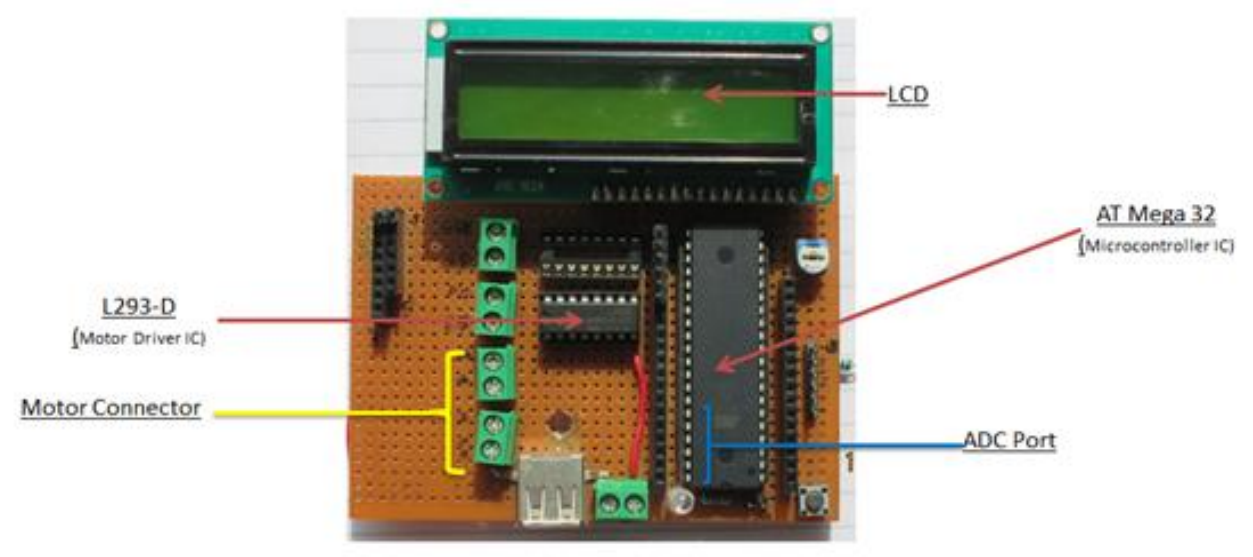

The ATmega32 is a low-power CMOS 8-bit microcontroller based on the AVR enhanced RISC architecture. At the core there are 32 general purpose working registers with rich instruction set. These registers are directly connected to Arithmetic Logic Unit (ALU), also allowing to access two independent registers in one single instrucion executed in one clock cycle.

It is 40pin PDIP with

- $32 \mathrm{~KB}$ of In-System Programmable Flash Program memory with READ-While-Write capabilities.

- 1024 bytes EEPROM

- $2 \mathrm{~KB}$ SRAM

- 32 general purpose working registers

- 32 general purpose I/O lines

- An 8-channel, 10 bit ADC

2.1 ADC conversion

- An ADC converts an input voltage into a number.

- An ADC has a resolution. A 10 Bit ADC has a range of 0-1023. (2^10=1024)

- The ADC also has a Reference voltage (ARef). When input voltage is GND the output is 0 and when input voltage is equal to ARef the output is 1023. So the input range is $\mathbf{0}$ to ARef and digital output is 0-1023.

\section{Remote control}

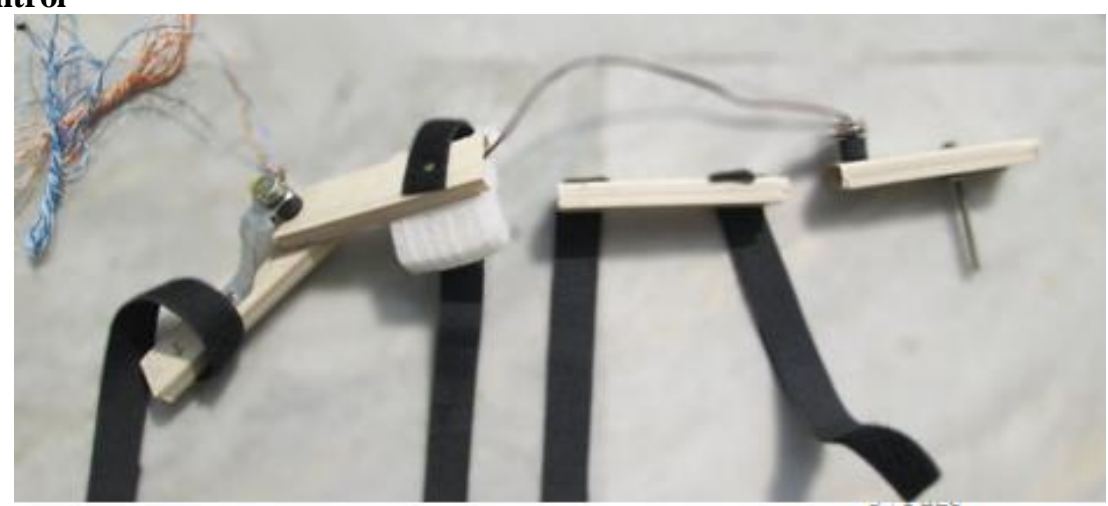

A sensor (also called detector) is a device that measures a physical quantity and converts it into equivalent electrical signal. The user in order to move the robotic arm should make a hand movement. This 
different movement is sensed by potentiometer attached to haptic gloves (Remote control). Potentiometer is used in haptic suit along with ADC for position feedback of joints. It gives the feedback in the form of voltage. The output of this sensors are in analog form, therefore they are connected to analog port of microcontroller AVR-ATmega32 (pin 39, 40).

\section{Figures And Tables}
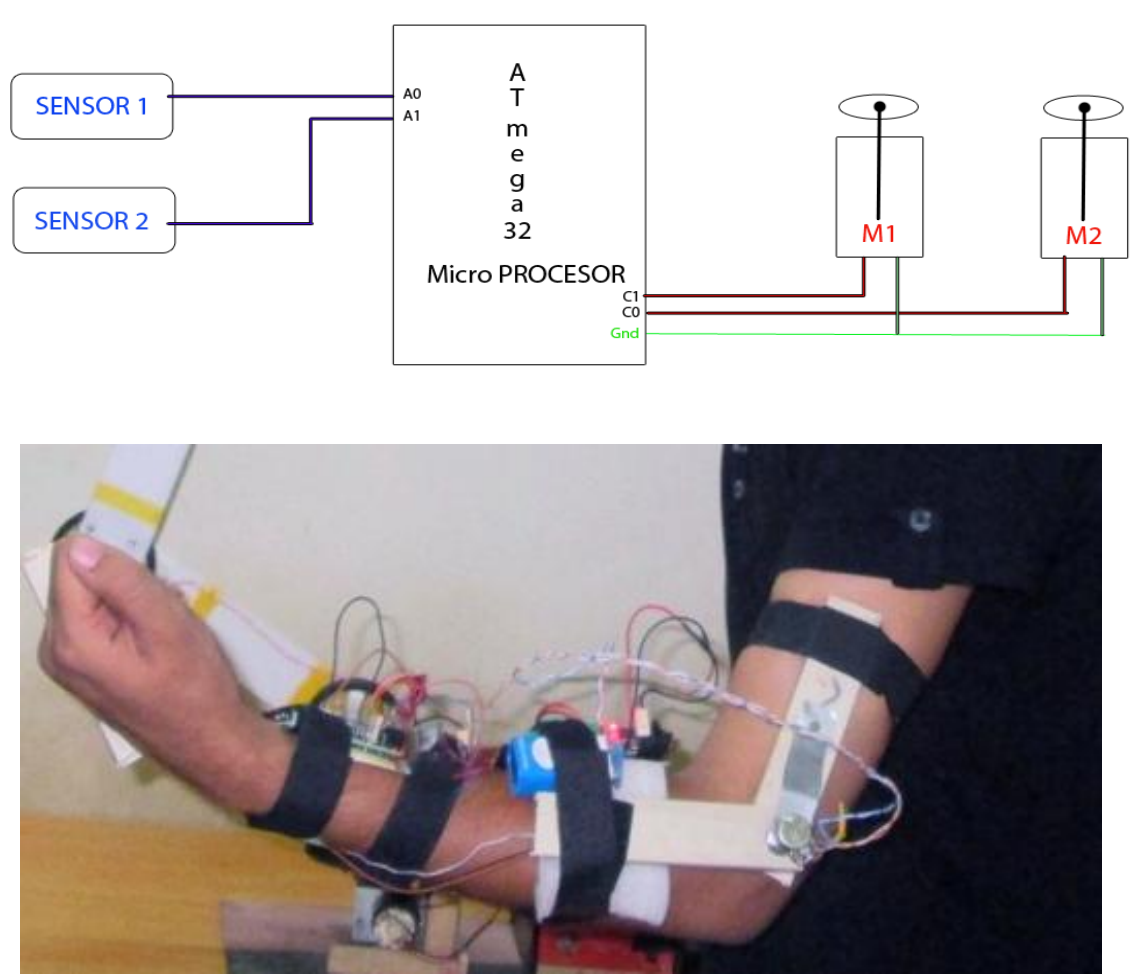

IV. Program Logic

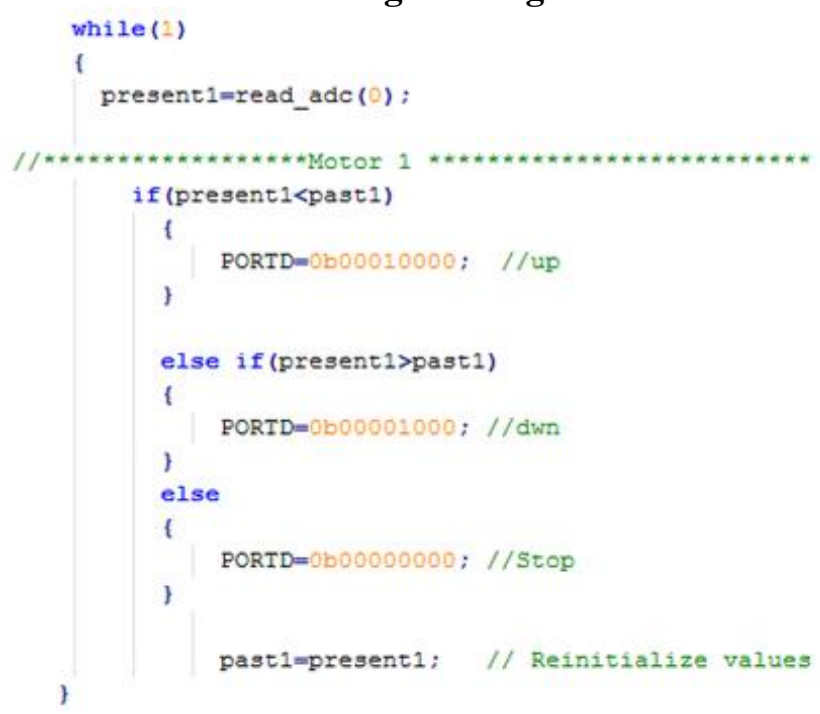

Programming is done in embedded c environment with eclipse IDE for AVR development. The main programming logic is based on acquiring the position of the arm and duplicating the motion of the human arm with robotic arm.

We take the input from the ADC port and convert to digital decimal value which is between 0 and 1023. This decimal value is stored in a variable "present1". Then it is compared with the previous stored value, if the current value is greater than the previous value, then it is moved in one direction else if less than the previous then move in other direction else no motion. After comparing the value present value is made past 
value and stored in variable "past1" and "present1" is reinitialized again. The whole loop continues for both the sensors and all the conditions are taken in account.

\section{Conclusion}

The robotic arm so far designed is able to lift the objects. In order to extend its capability, more advanced tools and material with the capacity to withhold the heavy weight objects are to be used, which is then applicable in warfront and used as a rescuer at several places where there is a need and also in industrial areas, military, and so on.

One important advancement can be use of smart haptic gloves which gives feedback of the object held by robotic arm so that operator can get the feel of operating with his own hand.

\section{Advantages}

- Can be applied in remote rural areas so as to carry out operations.

- Can be used in military areas where highly skilled doctors may not be present. In application like bomb disposal the human life is not at risk.

- It allows interactivity in real-time with virtual objects.

- Precise control of tools during operation.

- Reduction of no. of peoples needed in operation room

\section{Limitations}

- Debugging issues of these are complicated since they involve real-time data analysis.

- Links in telemedicine must have $0 \%$ fault rates.

- Auxiliary controls are required to move the workspace of the device to a new location.

- Full working of the robot is dependent on the range of RF transceiver.

\section{Future improvements}

- Stepper motors can be used for precise control and movement.

- Flex strips as sensors can be used in place of potentiometers to give more accurate input.

\section{References}

[1]. "Design And Implementation Of A Robotic Arm Based On Haptic Technology" Rama Krishna, G. SowmyaBala, A.S.C.S. Sastry, B. Bhanu Prakash Sarma, GokulSaiAlla / International Journal of Engineering Research and Applications (IJERA) ISSN: 22489622 www.ijera.com Vol. 2, Issue 3, May-Jun 2012, pp.3098-3103

[2]. "Robotic Arm" - www. NASA explores. com from Teacher Sheets pg:1-2

[3]. "ATMEL - short notes", www.atmel.com

[4]. "Six-servo Robot Arm" from www.arexx.com.cn on 13-11-2011 pg: 1-18

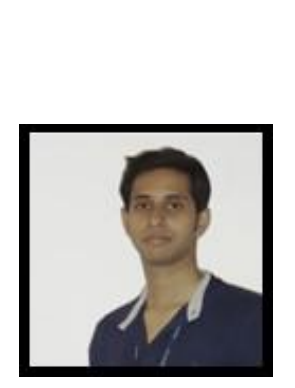

\section{About the Author}

Abhishek Verma,pursuing B. Tech degree in Computer Science and Engineering from BBD NITM, Lucknow. His research interests include Robotics, Artificial Intelligence and Machine Learning.

He can be contacted at abhiverma.abhishek@gmail.com

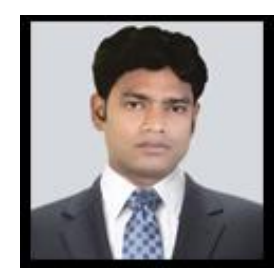

Ashish Sharma,has completed his B.Tech in Electronics Engineering from Dr. AITHCollege, Kanpur-India in 2009 andworking in R\&D department last five years. His research interests include Embedded Systems, Automation \& Robotics. He can be contacted at ashishpreet2009@ gmail.com 